Kavemann, Barbara (1998) Frauen als Täterinnen - Frauen, die Mädchen und Jungen sexuell missbrauchen, in: Wodtke-Werner, Verena (Hg.) Nicht wegschauen - Vom Umgang mit Sexualstraftätern, Weinheim, S. 31-44

Kavemann, Barbara (2003): Zur Debatte um Männer als Opfer und Frauen als Täterinnen häuslicher Gewalt. In: Frauenhauskoordinierung e.V. (Hrsg.): Sonderinfo 6 zum Aktionsplan der Bundesregierung zur Bekämpfung von Gewalt gegen Frauen. Frankfurt/M., S. 52-55.

Kavemann, Barbara (1997) Zwischen Politik und Professionalität: Das Konzept der Parteilichkeit, in: Hagemann-White, Carol; Kavemann, Barbara; Ohl, Dagmar: Parteilichkeit und Solidarität, Bielefeld, S. $179 \mathrm{ff}$

Kimmel, Michael S. (2002) "Gender Symmetry" in Domestic Violence. A substantive and methodological research review, Violence against Women Vol.8, Nr. 11 / 2002, pp 1332 $-1363$
Lenz, Hans-Joachim (Hg) (2000): Männliche Opfererfahrungen. Problemlagen und Hilfeansätze in der Männerberatung, Weinheim

Ohms, Constanze (1993) Mehr als das Herz gebrochen - Gewalt in lesbischen Beziehungen, Berlin

Piispa, Minna / Statistiks Finnland (2002) Complexity of Patterns of Violence Against Women in heterosexual Partnership, in: Violence against women Vol. 8 No. 4, pp. 873-900

Schröttle, Monika, Müller, Ursula; Glammeier, Sandra (2004) Lebenssituation, Sicherheit und Gesundheit von Frauen in Deutschland, BMFSFJ (Hg.) abzurufen unter www.bmfsfj. de/Forschungsnetz/Forschungsberichte

Silkenbeumer, Mirja (2007): Biografische Selbstentwürfe und Weiblichkeitskonzepte aggressiver Mädchen und junger Frauen. Münster, Lit Verlag.

Thürmer-Rohr, Christina (1989), Frauen in Gewaltverhältnissen, in: Studienschwerpunkt Frauenforschung TU-Berlin (Hg.)Mittäterschaft und Entdeckungslust, Berlin.
Tjaden, Patricia; Thoennes, Nancy (2000 a) Full Report of the Prevalence, Incidence and Consequences of Violence Against Women, National Institute of Justice, NCJ 183781, US Department of Justice

Tjaden, Patricia; Thoennes, Nancy (2000 b) Prevalence and consequences of male-to-female and female-to-male partner violence as measured by the National Violence Against Women Survey, Violence Against Women 6/2000, pp118-141

Die Autorin: Prof. Dr. Barbara Kavemann, Dipl. Soziologin, lehrt an der Katholischen Hochschule für Sozialwesen, Berlin und arbeitet seit 1978 zu Fragen der Gewalt im Geschlechterverhältnis und der sexualisierten Gewalt gegen Kinder und Jugendliche, vorwiegend in der Praxisevaluation und der Fortbildung.

\title{
Inhaftierte Frauen - eine aktuelle Bestandsaufnahme des Frauenstrafvollzugs in Deutschland
}

Ina Funk

\begin{abstract}
Möchte man sich im Rahmen einer wissenschaftlichen Arbeit mit dem Thema Frauenstrafvollzug befassen, so stößt man bereits bei der Suche nach geeigneter soziologischer und kriminologischer Fachliteratur an Grenzen. Nicht nur in quantitativer, sondern auch in qualitativer Hinsicht werden Missstände deutlich, denn es gibt kaum aktuelle Bestandsaufnahmen (Maelicke 1995, BAGS e.V. 1998, Zolondek 2007) zur Situation von weiblichen Inhaftierten. Dass Frauen als Bestrafte nur marginal in der Öffentlichkeit wahrgenommen werden, kann sowohl durch die geringe Medienpräsenz als auch durch deren schwache gesellschaftliche und sozialpolitische Beachtung bestätigt werden. Als eine mögliche Erklärung für das mangelnde öffentliche Interesse könnte sich in diesem Zusammenhang die niedrige Beteiligung der Frauen an der Gesamtkriminalität erweisen. Denn von den insgesamt am 31.03.2007 im Strafvollzug untergebrachten 75.719 Gefangenen waren lediglich 5,1 \% weiblichen Geschlechts. (vgl. Strafvollzugsstatistik 2007 a, S. 5) Hat die geringe Anzahl weiblicher Gefangener Auswirkungen auf den Frauenvollzug? Ist frauenspezifischer Vollzug überhaupt möglich?
\end{abstract}

Im Zuge einer wissenschaftlichen Arbeit beschäftigte ich mich 2007/2008 mit diesen Fragen und führte hierzu eine Untersuchung zur aktuellen Situation des Frauenstrafvollzugs in der Bundesrepublik durch. Die folgenden Ausführungen basieren auf den hieraus gewonnenen Erkenntnissen, die ich mit Hilfe einer Befragung der Justizministerien und Haftanstalten der einzelnen Bundesländer sowie der Auswertung aktueller Statistiken ermitteln konnte.

\section{Unterbringungsformen}

Frauen werden bundesweit in insgesamt 45 Haftanstalten inhaftiert. Bei lediglich sechs dieser Anstalten handelt es sich um eigenständige Frauenvollzugsanstalten. Diese befinden sich in den Bundesländern BadenWürttemberg (JVA Schwäbisch Gmünd), Bayern (JVA Aichach), Berlin (JVA für Frauen Berlin), Hessen (JVA Frankfurt am Main III), Niedersachsen (JVA für Frauen Vechta) und Nordrhein-Westfalen (JVA Willich II).

Am 31.03.2007 wurden dort 1.927 Frauen untergebracht. In Relation zu den insgesamt im Frauenstrafvollzug inhaftierten 4.068 weiblichen Gefangenen befanden sich dem- nach $47,4 \%$ in besonderen Frauenvollzugseinrichtungen.

Die zentrale Unterbringung in einer der wenigen selbständigen Haftanstalten bedeutet für viele Frauen, weit entfernt von ihrem Heimatort und damit weit weg von ihren Freunden und vor allem von ihrer Familie, ihren Ehemännern und Kindern leben zu müssen. Um den Kontakt aufrechtzuerhalten, sind Angehörige gezwungen, lange Anfahrtswege auf sich zu nehmen. Die damit verbunden Kosten können von vielen nicht oder nur selten getragen werden. (vgl. Kawamura-Reindl 2009, S. 356; Obermöller 1999, S. 135; Stöckle-Nicklas 1989, S. 113 f.; Zolondek 2007, S. 60) Dies kann langfristig zur Folge haben, dass soziale Beziehungen nach außen - vor allem bei Gefangenen, die eine lange Haftstrafe zu verbüßen haben - abbrechen und Frauen nach der Haft auf sich alleine gestellt sind. Die Integration in das „Leben in Freiheit" wird damit erheblich erschwert.

Auch können Vollzugslockerungen, die ebenfalls der Erhaltung von externen Kontakten und einer optimalen Entlassungsvorbereitung dienen sollen, nicht wahrgenommen werden. Denn selbst wenn sie hinsichtlich 
Abb1.: Belegung in eigenständigen Frauenvollzugsanstalten am 31.03.2007 nach Haftarten

\begin{tabular}{|c|c|c|c|c|c|c|c|}
\hline \multirow{2}{*}{$\begin{array}{c}\text { eigenständige } \\
\text { Frauenhaftanstalten }\end{array}$} & \multirow[b]{2}{*}{ U-Haft } & \multicolumn{2}{|c|}{ Freiheitsstrafe } & \multicolumn{2}{|c|}{ Jugendstrafe } & \multirow[b]{2}{*}{ Abschiebehaft } & \multirow[b]{2}{*}{$\begin{array}{l}\text { Belegung } \\
\text { insgesam }\end{array}$} \\
\hline & & $\begin{array}{l}\text { geschl. } \\
\text { Vollzug }\end{array}$ & $\begin{array}{l}\text { offener } \\
\text { Vollzug }\end{array}$ & $\begin{array}{l}\text { geschl. } \\
\text { Vollzug }\end{array}$ & $\begin{array}{l}\text { offener } \\
\text { Vollzug }\end{array}$ & & \\
\hline JVA Aichach & 41 & 388 & 5 & 58 & - & - & 492 \\
\hline JVA für Frauen Berlin & 58 & 97 & 78 & 19 & 5 & 2 & 259 \\
\hline JVA Frankfurt am Main III & 58 & 172 & 45 & 18 & 1 & 8 & 302 \\
\hline JVA Schwäbisch Gmünd & 57 & 290 & 5 & 33 & - & 7 & 392 \\
\hline JVA Vechta & 10 & 184 & 56 & 16 & 8 & - & 274 \\
\hline JVA Willich II & - & 151 & 57 & - & - & - & 208 \\
\hline insgesamt & 224 & 1.282 & 246 & 144 & 14 & 17 & 1.927 \\
\hline
\end{tabular}

nicht bestehender Flucht- oder Rückfallgefahr hierfür geeignet sind, verhindern weite Entfernungen und die ungünstige finanzielle Lage, in der sich viele weibliche Gefangene befinden, oftmals die Inanspruchnahme von Ausgang und Urlaub. (vgl. Grote-Kux 2007 b)

Obwohl das Strafvollzugsgesetz in $\$ 140$ Abs. 2 vorsieht, weibliche Gefangene getrennt von Männern in eigenen Frauenvollzugsanstalten unterzubringen und Frauen nur aus besonderen Gründen in Männervollzugseinrichtungen aufgenommen werden sollen, werden die meisten $(64,7 \%)$ - entgegen des Trennungsprinzips - entweder in separaten Abteilungen innerhalb von Männerhafthäusern, in eigenen Gebäuden innerhalb des Anstaltsgeländes bzw. außerhalb der Mauern (offener Vollzug) oder in Außenstellen, die ebenfalls an den Männervollzug angebunden sind, inhaftiert. ${ }^{1}$ Begründet wird dies damit, dass aufgrund der geringen Anzahl straffälliger Frauen nur wenige eigenständige Frauenhaftanstalten existieren können und dies - würden weibliche Gefangenen ausschließlich dort inhaftiert werden - für die meisten eine heimatferne Unterbringung zur Folge hätte. Festzustellen ist allerdings, dass auch die Zahl der Männervollzugsanstalten, in denen Frauen aufgenommen werden, nicht ausreichen, um den meisten die Möglichkeit einzuräumen, in der Nähe ihrer Familie und ihren Freunden inhaftiert zu sein. (vgl. Zolondek 2007, S. 60)

Erschwerend kommt hinzu, dass sie als „Anhängsel“ (Kawamura-Reindl 2009, S. 356) des Männervollzugs den selben, auf männliche Inhaftierte zugeschnittenen, strengen Sicherheitsvorkehrungen ausgesetzt sind, obwohl ihre Deliktstruktur, die vor allem von Eigentums- und Vermögensdelikten und weniger von schweren (Gewalt-)delikten geprägt ist, auf eine geringe Gefährlichkeit,
z.B. gegenüber Vollzugsbediensteten, schließen lässt. (vgl. Obermöller 1999, S. 93 f.) So befanden sich am 31.03.2007 22,3\% aller Frauen aufgrund eines Gewaltdeliktes ${ }^{2}$ und wegen eines Eigentums- und Vermögensdeliktes $^{3} 53,1 \%$ in Haft. Männer wurden dagegen zu 41,5\% aufgrund eines Gewaltdeliktes und nur zu 33,7 \% wegen eines Eigentums- und Vermögensdeliktes inhaftiert. (vgl. Strafvollzugsstatistik 2007 b, S. 22)

Auch die Entwicklung und Umsetzung eigener frauenspezifischer Behandlungskonzepte sowie Differenzierungen innerhalb der Insassenpopulation sind durch die Anbindung an den Männervollzug nur schwer zu realisieren. (vgl. Kawamura-Reindl 2009, S.356) So werden z.B. drogenabhängige gemeinsam mit nicht-drogenabhängigen und substituierten Frauen inhaftiert. Die Gefahr eines Rückfalls wird damit drastisch erhöht. Ebenso werden Gefangene, die bereits den Freigängerstatus erlangt haben, zusammen mit Inhaftierten, welche die Voraussetzungen noch nicht erfüllt haben, untergebracht. Dies kann zur Folge haben, dass Frauen, die die Haftanstalt verlassen dürfen, massiv unter Druck gesetzt werden, Botengänge für andere vorzunehmen. Gehen sie solchen Forderungen nicht nach, werden sie ausgegrenzt und zur Außenseiterin deklariert. (vgl. Jansen 1999, S. 25)

\section{Vollzugsdauer der verhängten Freiheitsstrafe}

Bei der Aufnahme in Haft soll im Rahmen einer Behandlungsuntersuchung - gemäß \$6 StVollzG - durch Erforschung der Persönlichkeit und Lebensumstände der Gefangenen in Erfahrung gebracht werden, welche Behandlungsmaßnahmen als sinnvoll und notwendig erachtet werden, um den Vollzug so zu gestalten, dass das Resozialisierungsziel „künftig in sozialer Verantwortung ein Leben ohne Straftaten zu führen“ ( $\mathbb{2}$ St-
VollzG) erreicht werden kann. Hierfür wird in aller Regel ein Vollzugsplan i.S.d. $\$ 7$ StVollzG erstellt. Er enthält neben Angaben über die Unterbringung im geschlossenen und offenen Vollzug, u.a. Angaben über Arbeits-, Ausbildungs- und Weiterbildungsmaßnahmen, besondere Hilfs- und Behandlungsmaßnahmen, Vollzugslockerungen und notwendige Maßnahmen zur Vorbereitung der Entlassung.

Von der Durchführung der Behandlungsuntersuchung und der damit verbundenen Erarbeitung des Vollzugsplans kann gemäß $\$ 6$ Abs. 1 Satz 2 StVollzG allerdings abgesehen werden, „wenn dies mit Rücksicht auf die Vollzugsdauer nicht geboten erscheint. “ Die Verwaltungsvorschrift des Strafvollzugsgesetzes sieht in diesem Zusammenhang eine Vollzugsdauer von bis zu einem Jahr vor.

Am 31.03.2007 lag die voraussichtliche Verweildauer bei 1.607 weiblichen Gefangenen, die sich aufgrund einer Freiheitsstrafe in Haft befanden, unter einem Jahr. Nur 1.307 Inhaftierte hatten eine Strafe von über einem Jahr zu verbüßen. (vgl. Strafvollzugsstatistik 2007 a, S. 6) Bei mehr als der Hälfte $(55,4 \%)$ war es demnach nicht erforderlich, eine Behandlungsuntersuchung vorzunehmen und einen Vollzugsplan zu entwickeln. Die Mehrheit aller Frauen wird somit lediglich verwahrt. (vgl. Kawamura-Reindl 2009, S. 357) Sie warten nur darauf, entlassen zu werden, zeigen kein Interesse am Anstaltsleben (vgl. Obermöller 2000, S.72) und setzen sich nicht mit sich und ihrer Lebenssituation auseinander.

\section{Belegung am 31.03.2007}

Am 31.03.2007 befanden sich bundesweit 4.068 Frauen in Haft. Ein Vergleich mit den Untersuchungen von Hannelore Maelicke und der Bundesgemeinschaft für Straffälligenhilfe, die ebenfalls die Situation des Frauenstrafvollzugs analysierten, zeigt eine 
kontinuierliche Zunahme der weiblichen Inhaftiertenzahlen in den letzten 14 Jahren. Wurden am 31.03.1993 lediglich 2.625 weibliche Gefangene gezählt, so waren es am 31.03.1997 bereits 3.097. (vgl. Maelicke 1995, S. 41; BAG-S e.V. 1998, S. 33) Insgesamt stieg die Insassenpopulation von 1993 bis 2007 somit um 55 \%.

In diesem Zusammenhang stellt sich die Frage, inwiefern die Justizvollzugsanstalten auf die gestiegenen Zahlen reagiert und demnach ihre Haftplatzkapazitäten erweitert haben. Der Strafvollzugsstatistik konnte entnommen werden, dass die am 31.03.2007 inhaftieren 4.068 weiblichen Gefangenen in ganz Deutschland auf 4.283 Haftplätze verteilt waren. Dies entspricht einer Kapazitätsauslastung von $95 \%$. Da unter Vollzugspraktikern nicht erst bei einer Auslastung von $100 \%$, sondern schon bei $85-90 \%$ von einer Vollbelegung gesprochen wird (vgl. Dünkel/Geng 2003, S. 146), wird deutlich, dass der Frauenvollzug bundesweit überbelegt war.

Eine besonders problematische Überbelegungssituation stellt sich in Sachsen (119,7 $\%)$, Baden-Württemberg (111,6 \%), Berlin $(107 \%)$, Bayern $(104,1 \%)$ und RheinlandPfalz $(100,5 \%)$ dar. ${ }^{4}$ Hier lagen die Belegungsquoten bei über $100 \%$. Geht man davon aus, dass der Vollzug bereits bei 90 $\%$ voll belegt ist, standen auch in Justizvollzugsanstalten von Sachsen-Anhalt (97,1\%) und Nordrhein-Westfalen (95,1\%) zu wenige Haftplätze zur Verfügung. In den Haftanstalten von Niedersachsen $(82,7 \%$ ), Hessen $(81,4 \%)$, Brandenburg $(78,6 \%)$ und Bremen $(77,1 \%)$ war dagegen eine Vielzahl von Haftplätzen unbesetzt. Die Bundesländer Hamburg und Schleswig-Holstein wiesen mit 62,9\% bzw. 61,9\% die geringsten Auslastungen auf. ${ }^{5}$

\section{Unterbringung im geschlossenen und offenen Vollzug}

Der geschlossene Vollzug war bundesweit zu 99,3\%, der offenen Vollzug dagegen nur zu 72,9\% belegt. Zwischen den einzelnen Bundesländern werden hierbei erhebliche Unterschiede deutlich:

Auffallend ist vor allem die Tatsache, dass in Bundesländern, in denen der geschlossenen Vollzug teilweise erheblich überbelegt ist (Bayern, Baden-Württemberg, Sachsen-Anhalt, Rheinland-Pfalz und Nordrhein-Westfalen), im offenen Vollzug eine Vielzahl von Kapazitäten ungenutzt bleibt. Mecklenburg-Vorpommern hält für weibliche Gefangene im offenen Vollzug keine Haftplätze bereit. Aber auch in den übrigen Bundesländern stehen den Frauen nur wenige Haftplätze zur Verfügung. So befanden sich von den insgesamt 4.068 weiblichen Inhaftierten am 31.03.2007 lediglich $513 \mathrm{im}$ offenen Vollzug. Der Anteil lag demnach bei
$12,6 \%$ und widerspricht damit „deutlich der Vorgabe des Strafvollzugsgesetzes $(\mathbb{\$} 10$ StVollzG), wonach der offenen Vollzug der Regelvollzug sein soll“ (Kawamura-Reindl 2009, S. 357). In Anbetracht dessen, dass weibliche Gefangene vor allem aufgrund von Eigentums- und Vermögensdelikten inhaftiert werden und weniger wegen Gewaltdelikten, bei denen es sich dann überwiegend um sog. „Konflikttaten im sozialen Nahraum“ (Obermöller 2000, S. 93) handelt und man daher von einer geringen Wiederholungsgefahr ausgehen kann, ist der Anteil weiblicher Gefangenen im offenen Vollzug zu gering. Auch weisen Statistiken über Vollzugslockerungen, wie Urlaub oder Ausgang, darauf hin, dass diese selten dafür missbraucht werden, um Fluchtversuche zu unternehmen oder weiter Straftaten zu begehen. (vgl. Obermöller 2000, S. 94) Dies spricht ebenfalls dafür, mehr Frauen in den offenen Vollzug zu verlegen.

Der offene Vollzug bietet die effektivste Möglichkeit, Inhaftierte auf ein Leben in Freiheit vorzubereiten. Denn hierdurch wird die Aufrechterhaltung der Kontakte zur Außenwelt erleichtert und die oft mit dem offenen Vollzug verbundene Gewährung von Lockerungen ermöglicht den Inhaftierten, die Anstalt zu verlassen, ihre Familie und Freunde zu besuchen oder einem freien Beschäftigungsverhältnis nachzugehen. (vgl. \11 StVollzG)

Tabelle2: Belegung und Belegungsfähigkeit im geschlossenen und offenen Vollzug am 31.03.2007

\begin{tabular}{|c|c|c|c|c|c|c|c|c|}
\hline \multirow{3}{*}{ Bundesland } & \multicolumn{4}{|c|}{ Belegung } & \multicolumn{2}{|c|}{ Belegungsfähigkeit } & \multicolumn{2}{|c|}{ Belegungsquote in $\%$} \\
\hline & \multicolumn{2}{|c|}{ geschl. Vollzug } & \multicolumn{2}{|c|}{ offener Vollzug } & \multirow{2}{*}{$\begin{array}{l}\text { geschl. } \\
\text { Vollzug }\end{array}$} & \multirow{2}{*}{$\begin{array}{l}\text { offener } \\
\text { Vollzug }\end{array}$} & \multirow{2}{*}{$\begin{array}{l}\text { geschl. } \\
\text { Vollzug }\end{array}$} & \multirow{2}{*}{$\begin{array}{l}\text { offener } \\
\text { Vollzug }\end{array}$} \\
\hline & Anzahl & in $\%$ & Anzahl & in $\%$ & & & & \\
\hline Baden-Württemberg & 475 & 99,0 & 5 & 1,0 & 418 & 12 & 113,6 & 41,7 \\
\hline Bayern & 812 & 99,1 & 7 & 0,8 & 769 & 18 & 173,1 & 38,9 \\
\hline Berlin & 176 & 68,0 & 83 & 32,0 & 160 & 82 & 110,0 & 102,2 \\
\hline Brandenburg & 55 & 83,3 & 11 & 16,7 & 63 & 21 & 87,3 & 52,4 \\
\hline Bremen & 30 & 81,1 & 7 & 18,9 & 42 & 6 & 71,4 & 116,1 \\
\hline Hamburg & 113 & 87,6 & 16 & 12,4 & 159 & 46 & 71,1 & 34,8 \\
\hline Hessen & 296 & 85,5 & 50 & 14,6 & 348 & 77 & 85,1 & 64,9 \\
\hline Mecklenburg-Vorpommern & 37 & 100,0 & - & - & 35 & - & 105,7 & - \\
\hline Niedersachsen & 240 & 79,5 & 62 & 20,5 & 268 & 97 & 89,6 & 63,9 \\
\hline Nordrhein-Westfalen & 754 & 80,6 & 181 & 19,4 & 770 & 224 & 97,9 & 80,8 \\
\hline Rheinland-Pfalz & 188 & 90,8 & 19 & 9,2 & 184 & 22 & 102,2 & 86,4 \\
\hline Saarland & 6 & 100,0 & - & - & 16 & - & 37,5 & - \\
\hline Sachsen & 239 & 83,6 & 47 & 16,4 & 195 & 44 & 122,6 & 106,8 \\
\hline Sachsen-Anhalt & 89 & 90,0 & 10 & 10,0 & 85 & 17 & 104,7 & 58,8 \\
\hline Schleswig-Holstein & 45 & 86,5 & 7 & 13,5 & 61 & 23 & 73,8 & 30,4 \\
\hline Thüringen & - & - & 8 & 100 & 6 & 15 & - & 53,3 \\
\hline insgesamt & 3.555 & 87,4 & 513 & 12,6 & 3.579 & 704 & 99,3 & 72,9 \\
\hline
\end{tabular}


Demnach kann davon ausgegangen werden, dass im Fall des Freiheitsentzugs der offene Vollzug in jeder Hinsicht die beste Alternative darstellt, um dem Vollzugsziel und den Gestaltungsgrundsätzen des $\ 3$ StVollzG Rechnung zu tragen.

Die Unterbringung im offenen Vollzug stellt sich zwischen den einzelnen Bundesländern sehr unterschiedlich dar: Während in Bayern und Baden-Württemberg die Anteile bei nur knapp $1 \%$ lagen, wurde in der JVA für Frauen in Berlin fast jede dritte Frau (32 \%) im offenen Vollzug untergebracht. In Niedersachsen (20,5\%), Nordrhein-Westfalen $(19,4 \%)$ und Bremen $(18,9 \%)$ konnte etwa jede Fünfte ihre Strafe im offenen Vollzug verbüßen. In den übrigen Bundesländern abgesehen von Mecklenburg-Vorpommern, dem Saarland und Thüringen ${ }^{6}$ - wurde der offene Vollzug zwischen 9,2 \% (Rheinland-Pfalz) und 16,7 \% (Brandenburg) aller weiblichen Gefangenen gewährt. Insgesamt stellt sich die Situation in den neuen Bundesländern etwas günstiger dar. In den alten Bundesländern wurden 87,8 \% im geschlossenen und $12,2 \%$ im offenen Vollzug inhaftiert. In den neuen Bundesländern betrug die Relation 84,7 \% zu 15,3\%. Dennoch bildet der offene Vollzug - entgegen der Vorschrift in $\ 10$ Abs. 1 StVollzG - in keinem Bundesland die Regelvollzugsform.

\section{Unterbringung im Jugendstrafvollzug}

Aufgrund einer Jugendstrafe befanden sich am 31.03.2007 insgesamt 381 weibliche Gefangene in Haft. In Relation zu allen Inhaftierten ergibt dies einen Anteil von 9,4 $\%$.

Trotz der beträchtlichen Zuwachsrate von 192,3 \% in den letzten 14 Jahren (am 31.03.1993 wurden nur 130 Mädchen im Jugendstrafvollzug gezählt), stellt die Gruppe der weiblichen inhaftierten Jugendlichen bzw. Heranwachsenden nach wie vor eine relativ kleine Einheit dar. Dies führt zu einer Vielzahl von Problemen und erheblichen Benachteiligungen junger Gefangener.

In der Regel sind Mädchen - entgegen der Forderung des Jugendgerichtgesetzes, die Jugendstrafgefangene in eigene Haftanstalten zu inhaftieren - aufgrund ihrer geringen Anzahl in kleinen abgetrennten Abteilungen innerhalb des Frauenvollzugs untergebracht. (vgl. Grote-Kux 2007 b) Lediglich in Mecklenburg-Vorpommern werden junge Frauen seit Januar 2008 in der Jugendanstalt Neustrelitz aufgenommen. ${ }^{7}$ Die übrigen befinden sich in den fünf eigenständigen Frauenvoll- zugsanstalten Aichach (Bayern), Schwäbisch Gmünd (Baden-Württemberg), Berlin, Frankfurt am Main III (Hessen) und Vechta (Niedersachen) sowie in den Haftanstalten Köln (Nordrhein-Westfalen), Zweibrücken (Rheinland-Pfalz), Chemnitz (Sachsen) und Halle I (Sachsen-Anhalt). Weibliche Jugendstrafgefangene aus anderen Bundesländern werden im Rahmen von Vollzugsgemeinschaften dorthin verlegt. Demnach sind überwiegend junge Gefangene von der heimatfernen Unterbringung und der Trennung von Familie und Freunden betroffen, was vor allem von ihnen als besonders belastend empfunden wird.

Durch die Einbindung in den Erwachsenenvollzug sind die straffällig gewordenen Mädchen weiteren Nachteilen - gegenüber ihren männlichen Altergenossen, denen eigenständige Jugendanstalten zur Verfügung stehen - ausgesetzt. Dem vom Jugendgerichtsgesetz vorgeschriebenem Erziehungsauftrag (vgl. \91 JGG) kann nicht in dem Maße Rechnung getragen werden wie angedacht (vgl. Grote-Kux 2007 b), auf jugendspezifische, „alters- und entwicklungsbedingte Erfordernisse" (Walter 2003, S.10) kann nicht in gleicher Weise eingegangen werden. Denn „es gibt kaum Angebote für Bildung, Therapie [und] Beratung “ (Jansen 1999, S. 19), die auf die besonderen Bedürfnisse junger (weiblicher) Gefangener ausgerichtet sind.

Eine weitere strukturelle Benachteiligung erfahren sie bei der Unterbringung im offenen Vollzug. Ebenfalls aufgrund der geringen Gefangenenzahlen ist in nur wenigen Haftanstalten (Berlin, Frankfurt am Main III, Vechta, Köln und Chemnitz), in denen Jugendvollzug möglich ist, eine unabhängige und vom Erwachsenenvollzug abgetrennte Abteilung für jugendliche Strafgefangene eingerichtet. Am 31.03.2007 befanden sich daher nur $8 \%$ aller inhaftierten Mädchen im offenen Vollzug. Im erwachsenen Frauenstrafvollzug lag der Anteil immerhin bei $20 \%$.

\section{Arbeits- und Ausbildungsmöglich- keiten}

Im Hinblick auf die im Vollzug bestehenden Arbeits- und Ausbildungsmöglichkeiten können ebenfalls Benachteiligungen für weibliche Gefangene konstatiert werden. Die aktuelle Befragung der Justizministerin und Haftanstalten ergab, dass sich in eigenständigen Frauenvollzugsanstalten die Situation etwas günstiger darstellt. Denn aufgrund der hohen Inhaftiertenzahlen und der daraus resultierenden Größe der Haftanstalt können weiblichen Gefangenen breitere und auf ihre Bedürfnisse besser abgestimmte Bildungs- und Arbeitsmaßnahmen geboten werden. Vor allem bei der Unterbringung in kleinen Männeranstalten erweist sich die Lage allerdings nach wie vor als problematisch: Ausbildungsmöglichkeiten stehen den dort inhaftierten Frauen in den seltensten Fällen zur Verfügung. Ebenso reichen die Arbeitsplätze meist nicht aus, um allen Frauen eine berufliche Tätigkeit zuzuweisen. Infolgedessen können kaum Rücklagen gebildet werden, die der Tilgung von Schulden, mit denen sich viele Gefangene konfrontiert sehen, dienen können und die Wiedereingliederung in die Gesellschaft erleichtern. (vgl. Zolondek 2007, S. 232)

Darüber hinaus sind die Chancen auf dem Arbeitsmarkt mit den angebotenen Lehrberufen sehr gering. So können sich Frauen beispielsweise in der niedersächsischen JVA Vechta nur zur Köchin ausbilden lassen. In der JVA Köln werden lediglich Ausbildungen zur Friseurin, Textilreinigerin und Modenäherin angeboten. Gelingt es Frauen nach ihrer Entlassung dennoch in diesen meist traditionell schlecht bezahlten Frauenberufen eine Anstellung zu finden, reicht das geringe Gehalt selten aus, um sich und ggf. ihre Familie zu versorgen.

Ebenso können die meisten beruflichen Tätigkeiten, die sich vorwiegend auf einfache Montier-, Verpackungs- und Reinigungsarbeiten beschränken, weibliche Inhaftierte nicht ausreichend qualifizieren, um ihnen ein finanziell gesichertes Leben außerhalb der Haft zu ermöglichen.

Einer Vielzahl von Frauen bleibt auch deshalb die Teilnahme an Ausbildungsangeboten verwehrt, weil ihre Haftzeit zu kurz ist, um die Bildungsmaßnahme während der Inhaftierung abzuschließen. (vgl. Franze 2001, S. 298 f.) Entgegengewirkt wird dieser Problematik in einigen Anstalten, indem verschiedene Anqualifizierungsmaßnahmen mit oder ohne Zertifizierung angeboten werden. So können Insassinnen der JVA Luckau-Duben in Brandenburg beispielsweise den PC-Führerschein erwerben. In der nordrhein-westfälischen JVA BielefeldBrackwede II haben weibliche Gefangene die Möglichkeit, an Qualifizierungsmaßnahmen im Gartenbau teilzunehmen und in der JVA Bützow (Mecklenburg-Vorpommern) werden Frauen an die Tätigkeiten eines CallCenter-Agents herangeführt.

Im Hinblick auf berufliche Beschäftigungen ist zudem darauf hinzuweisen, dass von ih- 
nen abgesehen werden kann, wenn dies im Hinblick auf die Vollzugsdauer nicht geboten erscheint. (vgl. $\$ 6$ Abs. 1 Satz 2 i.V.m. \ 7 Abs. 2 Nr. 4 StVollzG)

Es kommt erschwerend hinzu, dass weibliche Gefangene aufgrund ihres oftmals geringen Bildungsniveaus für eine Reihe von Maßnahmen nicht geeignet sind. Frustration und Überforderung und sogar Arbeitsverweigerung können die Folge sein. Ebenso kann sich der Mangel an Motivation und Interesse als Grund für die Untätigkeit erweisen. (vgl. Zolondek 2007, S 234 f.)

Für Frauen mit Vollzugslockerungen stellt sich die Ausbildungs- und Arbeitssituation am günstigsten dar. Im Rahmen des

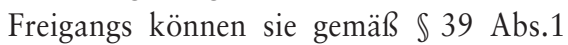
StVollzG einem freien Ausbildungs- oder Beschäftigungsverhältnis nachgehen. Die Entlohnung beruflicher Tätigkeiten richtet sich dabei nicht nach dem niedrigen Arbeitsentgelt der Haftanstalten, sondern nach den Einkommensrichtlinien des Arbeitgebers. Die Lebensverhältnisse können somit am besten an diejenigen in Freiheit angepasst werden. Es dient dem Wiedereingliederungsprozess und folgt damit dem Resozialisierungsgedanken. In der Praxis zeigt sich allerdings, dass den wenigsten weiblichen Gefangenen Vollzugslockerungen gewährt werden. Die von Juliane Zolondek durchgeführte Untersuchung verdeutlicht dies: 2006 wurde lediglich einer Inhaftierten der JVA Bützow (Mecklenburg-Vorpommern) Freigang gestattet, in der Haftanstalt Berlin waren es zwei. Die ebenfalls befragte eigenständige Frauenvollzugsanstalt Vechta und deren Teilanstalt Hildesheim konnte sogar keine einzige Frau mit Freigängerstatus vorweisen. (vgl. Zolondek 2007, S. 256)

\section{Hilfeangebote}

Die Inhaftierung wird besonders von Frauen als äußerst belastend empfunden. Sie leiden weitaus mehr als die meisten Männer unter der Trennung von ihrer Familie, machen sich große Sorgen um ihre Kinder, entwickeln Schuldgefühle und haben mit starken Versagensängsten zu kämpfen. (vgl. Geiger/Steinert 1993, S. 60) Hinzu kommt die Stigmatisierung und moralische Verurteilung durch Freunde und Verwandte, die durchaus häufiger gegenüber weiblichen Gefangenen zum Tragen kommen. Sie müssen vermehrt die Erfahrung machen, dass sich ihr gesamtes soziales Umfeld, sogar der (Ehe-)Partner von ihnen abwendet. Werden Frauen nach der Verbüßung ihrer Haftstrafe entlassen, sind kaum Kontakte vorhanden, die ihnen bei der Wiedereingliederung in die Gesellschaft helfend und unterstützend zur Seite stehen können.

Weitere frauenspezifische Problemlagen finden sich bei der Betrachtung ihrer vergangenen und gegenwärtigen Lebenssituationen. Der Großteil weiblicher Inhaftierter wurde in früherer Kindheit Opfer von Gewalt und sexuellen Übergriffen. Viele dieser Frauen begeben sich auch oder sogar deswegen in Beziehungen, in denen sie erneut Gewalt und Demütigung erfahren. Sie unterwerfen und fügen sich ihrem Mann. Abhängigkeit, Unselbstständigkeit, mangelndes Selbstwertgefühl, Minderwertigkeitskomplexe und Schamgefühle können die Folge sein. (vgl. Maelicke 1995, S. 90)

Auf die Bedingungen in Haft reagieren sie daher in weit größerem Maße als Männer mit Anpassung, Passivität oder Resignation, Depressionen, Krankheit, Suizidversuch oder mit dem Konsum von (illegalen) Drogen. (vgl. Grote-Kux 2007 a; KawamuraReindl 2009, S. 350 ff.)

Hilfeangebote müssen demnach darauf abzielen, Frauen bei der Aufarbeitung ihrer traumatischen Erfahrungen und beim Erlernen von Bewältigungsstrategien zu unterstützen. (vgl. Geiger/Steinert 1993, S. 51; S. 61) Gefördert werden sollen Eigenständigkeit und Selbstverantwortung. Sie sollen befähigt werden, ein fundiertes Selbstbewusstsein zu entwickeln, sich und ihre Lebenssituation zu reflektieren und nach der Entlassung ein straf- und vor allem drogenfreies Leben zu führen. (vgl. BAG-S e.V. 1998, S. 55) Die Hilfe zur Selbsthilfe soll dabei immer im Vordergrund stehen.

Die Tätigkeiten der Sozialen Dienste im Vollzug, deren Einsatz durch die $\mathbb{S} 71$ bis 74 und $\$ 7$ Abs. 7 Nr. 6, 8 StVollzG geregelt ist, sollen gemäß \154 Abs. 2 Satz 1 StVollzG durch Angebote externer Fachkräfte verschiedenster Beratungs- und Betreuungsstellen ergänzt werden. Darüber hinaus sieht das Strafvollzugsgesetz in $\$ 154$ Abs. 2 Satz 2 die Zusammenarbeit von „Vollzugsbehörden [...] mit Personen und Vereinen vor, deren Einfluss die Eingliederung des Gefangenen fördern kann.“

Welche vollzugsinternen Beratungs- und Betreuungsangebote weiblichen Gefangenen zur Verfügung stehen und inwieweit den Forderungen des Strafvollzugsgesetzes Rechnung getragen wird, sollte ebenfalls durch die bundesweiten Befragung der Jus- tizministerien und Haftanstalten in Erfahrung gebracht werden.

Die Auswertung der Ergebnisse gestaltete sich allerdings schwierig, denn die gestellten Fragen wurden relativ unterschiedlich und zum Teil unvollständig oder ungenau beantwortet. So wurden für einige Vollzugseinrichtungen kaum Angaben zu Zahlen interner Fachkräfte oder ehrenamtlicher Mitarbeiter gemacht. Auch die genaue Ausgestaltung sozialer Hilfe im Vollzug blieb bei vielen Haftanstalten offen. Inwiefern es sich bei Unterstützungsangeboten um frauenspezifische Hilfeangebote handelt und ob Mitarbeiter der internen und externen Fachdienste sowie ehrenamtliche Betreuer ausschließlich den Frauen vorbehalten sind, konnte ebenfalls nur vereinzelt in Erfahrung gebracht werden.

Deutlich wird durch die Befragung jedoch, dass die Hilfeangebote für inhaftierte Frauen landesweit sehr unterschiedlich gestaltet sind und in den meisten Haftanstalten nicht ausreichen. Dies betrifft vor allem weibliche Gefangene, die sich in kleine Abteilungen innerhalb von Männervollzugsanstalten befinden, denn dort stehen die Fachkräfte meist nicht ausschließlich Frauen zur Verfügung. Demnach kann davon ausgegangen werden, dass eine auf weibliche Problemlagen ausgerichtete soziale Unterstützung für Frauen in Männervollzugseinrichtungen nur selten gewährleistet werden kann. In eigenständigen Haftanstalten hingegen stellt sich die Situation günstiger dar. Das Personal wird speziell - an den Bedürfnissen weiblicher Inhaftierter orientiert - geschult. Darüber hinaus bieten auch die in der Nähe von selbstständigen Frauenanstalten befindlichen externen Beratungs- und Betreuungsstellen vermehrt frauenspezifische Hilfe an. Frauen in Vollzugseinrichtungen, die in Großstädten angesiedelt sind, können ebenfalls von besseren und umfangreicheren Angeboten der freien Straffälligenhilfe profitieren.

Unterschiede zwischen den Bundesländern werden auch bei der Stellenbesetzung vollzugsinterner Sozialer Dienste deutlich. In der JVA Aichach werden beispielsweise knapp 500 weibliche Inhaftierte von lediglich sechs Sozialpädagoginnen betreut. Die Fallbelastung einer Sozialarbeiterin liegt demnach bei 83 Gefangenen. In der niedersächsischen JVA Vechta sind dagegen 14 Sozialpädagogen für 270 Gefangene zuständig und in der JVA Berlin wird die vollzugsinterne soziale Unterstützung der 262 Frauen von acht Sozialarbeitern geleistet. 
Dennoch sind in den meisten Haftanstalten, in denen Frauen untergebracht werden, die Fachdienste unzureichend besetzt. Es stellt sich demnach die Frage, inwieweit das Fachpersonal aufgrund seines umfangreichen Tätigkeitsprofils - denn die Arbeit beinhaltet neben der sozialen Unterstützung der Gefangenen auch Verwaltungstätigkeiten, wie z.B. das Verfassen von Berichten und Stellungnahmen - intensive Betreuung und somit effektive Hilfe leisten kann. (vgl. Obermöller 1999, S. 102 f.)

So erscheint es umso wichtiger, externe Mitarbeiter zu beschäftigen und ehrenamtliche Helfer zu finden, die das Personal entlasten. „Außerdem haben vollzugsfremde Personen eher die Möglichkeit, das erforderliche Vertrauensverhältnis zu Gefangenen aufzubauen “ (Obermöller 1999, S.129). Darüber hinaus können den straffällig gewordenen Frauen nur von diesen nach ihrer Haftentlassung weiterführende Unterstützungsmöglichkeiten angeboten werden.

Das ehrenamtliche Engagement gestaltet sich in den einzelnen Haftanstalten sehr unterschiedlich und ist in einigen Justizvollzugsanstalten ebenfalls noch ausbaufähig.

\section{Sozialtherapie}

Eine besondere Form vollzugsinterner Hilfe stellt die sog. Sozialtherapie dar. Gemäß $\ 9$ Abs. 1 StVollzG ist ein Gefangener in einer sozialtherapeutischen Anstalt unterzubringen, wenn er aufgrund eines Sexualdeliktes ( $\$ \int 174-180$ oder 182 StGB) eine zeitige Freiheitsstrafe von mehr als zwei Jahren zu verbüßen hat. Ferner können Inhaftierte, die nicht zur Gruppe der Sexualstraftäter gehören, einen (freiwilligen) Antrag auf
Verlegung stellen, sofern die besonderen therapeutischen Mittel und sozialen Hilfeangebote der Anstalt ihrer Resozialisierung dienen können. Hierüber hat der Leiter der Sozialtherapie zu entscheiden (vgl. $\$ 9$ Abs.2 StVollzG).

Bundesweit gibt es 47 sozialtherapeutische Einrichtungen. Für weibliche Strafgefangene stehen allerdings nur drei Abteilungen zur Verfügung. Diese befinden sich in den Justizvollzugsanstalten Berlin-Neukölln ${ }^{8}$ und Dresden (Sachsen) sowie in der Teilanstalt Alfeld der JVA Vechta (Niedersachsen). Insgesamt existieren dort 37 Haftplätze 9 . (vgl. Spöhr 2007, S. 34 f.) Allerdings wurde bereits 2003 der Bedarf auf 127 Haftplätze geschätzt. (vgl. Rehn 2003, S. 68) Am 31.03.2007 befanden sich 32 Frauen in den ihnen vorbehaltenen sozialtherapeutischen Abteilungen $^{10}$. (vgl. Spöhr 2007, S. 8) Dies entspricht einem Anteil von 1,1 \% an allen zu einer Freiheitsstrafe verurteilten weiblichen Gefangenen. Verlegt wird demnach nur ein Bruchteil aller strafgefangener Frauen. Weibliche Inhaftierte, denen eine Aufnahme gewährt wird, sehen sich dagegen aufgrund der wenig vorhandenen sozialtherapeutischer Abteilung mit dem Problem der heimatfernen Unterbringung konfrontiert.

Die geringe Anzahl der dort inhaftierten weiblichen Gefangenen kann u.a. auf die zu verbüßende Haftzeit zurückgeführt werden, denn eine effektive Behandlung ist in der Regel erst nach $1 \frac{112}{2}$ Jahren zu erwarten. (vgl. Drenkhahn 2007, S. 171) Da allerdings über die Hälfte aller Frauen zu kürzeren Freiheitsstrafen verurteilt werden, bleibt den meisten die Verlegung in eine sozialtherapeutische Abteilung verwehrt.
Eine weitere Erklärung findet sich bei der Betrachtung der für die Inhaftierung maßgeblichen Straftaten. Die verpflichtende Unterbringung in eine sozialtherapeutische Einrichtung für Sexualstraftäter mit einer Freiheitsstrafe von mehr als zwei Jahren gemäß $\int 9$ Abs. 1 StVollzG erfolgte lediglich bei neun der 32 Frauen (28,1 \%). Bei Männern lag der Anteil bei $60 \%$. Die Mehrheit aller weiblichen Gefangenen (71,9\%) wurde daher zu Delikten verurteilt, die unter $\$ 9$ Abs. 2 StVollzG zu fassen sind und bei denen es der Antragsstellung zur Verlegung bedarf. Den Einrichtungen steht es daraufhin frei, der Aufnahme zuzustimmen.

Die einzige offene Sozialtherapie für Frauen wird in der JVA Dresden geführt. Demnach wurde nur jede fünfte Frau $(21,9 \%)$, die sich am 31.03.2007 in sozialtherapeutischer Behandlung befand, im offenen Vollzug untergebracht. (vgl. Spöhr 2007, S. 36)

\section{Besonderheiten des Frauenstrafvollzugs}

Basierend auf $\$ 80$ StVollzG ist es weiblichen Gefangenen unter bestimmten Voraussetzungen gestattet, ihre Haftstrafe gemeinsam mit ihren nicht schulpflichtigen Kindern in speziellen Mutter-Kind Einrichtungen zu verbüßen. Vor allem wenn der Vater oder andere Familienangehörige die Versorgung des Kindes nicht oder nur unzureichend sicherstellen können und die Inhaftierung der Mutter eine Fremdunterbringung in Heimen oder Pflegefamilien zur Folge hätte, soll vom zuständigen Jugendamt ( $\int 80$ Abs. 1 Satz 2 StVollG) geprüft werden, ob eine gemeinsame Aufnahme erfolgen kann. Hierdurch soll möglichen Entwicklungsdefiziten und -störungen des Kindes aufgrund der Tren-

Abb. 3: Mutter-Kind-Einrichtungen

\begin{tabular}{|c|c|c|c|c|c|}
\hline \multirow{2}{*}{ Bundesland } & \multirow{2}{*}{ Justizvollzugsanstalt } & \multirow{2}{*}{ Unterbringungsform } & \multirow{2}{*}{$\begin{array}{l}\text { Anzahl der } \\
\text { Haftplätze }\end{array}$} & \multicolumn{2}{|c|}{ Belegung am 31.03.2007 } \\
\hline & & & & Mütter & Kinder \\
\hline Baden-Württemberg & Schwäbisch Gmünd ${ }^{11}$ & halboffener Vollzug ${ }^{12}$ & 11 & 8 & 10 \\
\hline Bayern $^{13}$ & Aichach & geschlossener Vollzug & 16 & 10 & 10 \\
\hline Berlin & $\begin{array}{l}\text { Berlin, Bereich Pankow } \\
\text { Berlin, Bereich Neukölln } \\
\text { Berlin, Bereich Reinickendorf }\end{array}$ & $\begin{array}{l}\text { geschlossener Vollzug } \\
\text { offener Vollzug } \\
\text { offener Vollzug }\end{array}$ & $\begin{array}{l}2 \\
3 \\
1\end{array}$ & $\begin{array}{l}- \\
2 \\
1\end{array}$ & $\begin{array}{l}- \\
2 \\
1\end{array}$ \\
\hline Hessen & $\begin{array}{l}\text { Frankfurt am Main III } \\
\text { Frankfurt am Main III }\end{array}$ & $\begin{array}{l}\text { geschlossener Vollzug } \\
\text { offener Vollzug }\end{array}$ & $\begin{array}{r}5 \\
18 \\
\end{array}$ & $\begin{array}{l}5 \\
5\end{array}$ & $\begin{array}{l}5 \\
5\end{array}$ \\
\hline Niedersachsen & $\begin{array}{l}\text { Vechta, Hauptanstalt } \\
\text { Vechta, Abteilung Falkenrott }\end{array}$ & $\begin{array}{l}\text { geschlossener Vollzug } \\
\text { offener Vollzug }\end{array}$ & $\begin{array}{r}5 \\
13\end{array}$ & $\begin{array}{r}2 \\
13\end{array}$ & $\begin{array}{r}2 \\
16\end{array}$ \\
\hline Nordrhein-Westfalen & JVK Fröndenberg & offener Vollzug & 16 & 16 & 18 \\
\hline Sachsen & Chemnitz, Bereich Reichenhain & offener Vollzug & 5 & 4 & 4 \\
\hline insgesamt & & & 95 & 66 & 73 \\
\hline
\end{tabular}


nung von der Bezugsperson, die in aller Regel die Mutter darstellt und besonders in den ersten Lebensjahren von großer Bedeutung ist, entgegengewirkt werden. (Zolondek 2007, S. 68 ff.; Kawamura-Reindl/von den Driesch 2002, S. 182; Franze 2001, S. $226 \mathrm{ff}$.)

Birtsch und Rosenkrank kamen 1988 bei einer Untersuchung zu der Erkenntnis, dass sich - trotz anhaltender Kritik, die Kinder seien der Gefahr der Prisonisierung ausgesetzt und könnten als „Vehikel der Resozialisierung der Mutter" (Quensel/Dürkop 1990, zit. nach Simmedinger 1995, S. 207) missbraucht werden - die gemeinsame Unterbringung oftmals als „die bessere Alternative zur Trennung von der Mutter“ erweist und vor allem „der Abwendung von Schlimmerem “ dient (Birtsch/Rosenkrank 1988, S. 201)

Im gesamten Bundesgebiet existieren sieben Mutter-Kind Einrichtungen mit insgesamt 95 Haftplätzen (Stand: 31.03.2007). Entsprechend der Empfehlung des $\$ 142$ StVollzG befinden sich diese vor allem in den Frauenvollzugsanstalten Schwäbisch Gmünd, Aichach, Berlin, Frankfurt am Main III und Vechta. Nordrhein-westfälische weibliche Gefangene können mit ihren Kindern in der im Justizvollzugskrankenhaus Fröndenberg eingerichteten Mutter-Kind-Abteilung untergebracht werden und in Sachsen werden straffällig gewordenen Frauen und deren Kinder gemeinsam in der JVA Chemnitz, Bereich Reichenhain, inhaftiert.

Am 31.03.2007 befanden sich insgesamt 66 Mütter mit 73 Kindern in Haft. Hinsichtlich der zur Verfügung stehenden Haftplätze entspricht dies einer Auslastung von knapp 70 $\%$.

Die geringe Auslastung ist allerdings nicht darauf zurückzuführen, dass kein Bedarf besteht, sondern dass für die meisten Frauen aufgrund der heimatfernen Unterbringung eine Verlegung in eine Mutter-Kind Einrichtung nicht in Betracht kommt. Darüber hinaus können die Aufnahmekriterien oftmals nicht erfüllt werden. So darf gemäß $\mathbb{8} 80$ Abs. 1 Satz 1 StVollG das Alter des Kindes das sechste Lebensjahr (bis zur Einschulung) nicht überschreiten. Denn man geht davon aus, dass Kinder die Bedingungen der Haft umso bewusster erleben, je älter sie werden. Es besteht die Gefahr, dass sie sich selbst eingesperrt fühlen. Auch die durch die Inhaftierung entstehende psychische Belastung der Mutter wird mit zunehmendem Alter direkter wahrgenommen. Dies könnte sich ebenfalls negativ auf die Entwicklung der Kinder auswirken.

In der Praxis zeigt sich allerdings, dass eine Aufnahme bis zum sechsten Lebensjahr lediglich in einigen wenigen Mutter-Kind Abteilungen (JVA Frankfurt am Main, JVA Vechta, JVA Fröndenberg), bei denen es sich zudem um offene Vollzugsformen handelt, erfolgt. In den übrigen Einrichtungen variiert die Höchstaltersgrenze der Kinder zwischen einem (JVA Berlin, Standort Pankow) und vier Jahren (JVA Aichach). ${ }^{14}$

Eine weitere Besonderheit des Frauenstrafvollzugs stellt der sog. Hausfrauenfreigang dar. Dabei ist es Frauen, die sich im offenen Vollzug befinden, im Rahmen des Freigangs gem. \11 Abs. 1 Nr.1 StVollzG gestattet, tagsüber - sofern die Versorgung nicht durch andere Personen sichergestellt werden kann - nach Hause zu fahren, um dort für ihre Kinder zu sorgen.

Ebenso wie durch Mutter-Kind-Einrichtungen soll auch durch den Hausfrauenfreigang einer Fremdunterbringung der Kinder in Heimen oder Pflegefamilien entgegengewirkt werden. Ob sich dies aber als die optimale Lösung erweist, ist fraglich, denn „die organisatorisch wie persönlich meist sehr anstrengende und belastende Realisierung des Hausfrauenfreigangs kann das Erproben von Selbstständigkeit und Unabhängigkeit erschweren “ (Kawamura-Reindl 2009, S. 359), die Frauen verbleiben daher oftmals in der ihr zugeschriebenen klassischen Rolle der Hausfrau und Mutter.

Letzten Endes stellt der Hausfrauenfreigang somit lediglich die bessere Alternative zur gemeinsamen Inhaftierung oder Fremdunterbringung der Kinder dar.

2007 wurde dieser 33 Frauen gestattet. Die praktische Relevanz erweist sich demnach als äußerst gering. Dies ist vor allem darauf zurückzuführen, dass die Entfernungen zwischen der Haftanstalt und dem Heimatort so groß sind, dass sie innerhalb eines Tages nicht zurückgelegt werden können. Zudem müssen die Fahrtkosten von den Frauen selbst getragen werden, was oftmals durch die schlechte finanzielle Lage, in der sich viele weibliche Inhaftierte befinden, nicht möglich ist. Es stellt sich demnach die Frage, ob bei Frauen, die die Voraussetzungen des Hausfrauenfreigangs erfüllen, nicht eine vorzeitige Haftentlassung, ggf. im Gnadenweg, in Betracht gezogen werden kann. (vgl. Kawamura-Reindl 2009, S. 359)

\section{Schlusswort}

Die seit Jahren in der wenigen Fachliteratur bestehende Annahme „Frauenstrafvollzug ist Männersache“ (Maelicke 1995, S.115) lässt sich durch die Ausführungen erneut bestätigen. Trotz der erheblichen quantitativen Zunahme ${ }^{17}$ der weiblichen Gefangenenpopulation in den vergangenen Jahren hat sich an der defizitären Lage kaum etwas verändert. so dass man sich fragen muss, wann die kritische Menge inhaftierter Frauen erreicht ist, um dieser Lage angemessen sozialpolitisch zu begegnen.

Abb. 4: Hausfrauenfreigang 2007

\begin{tabular}{|l|l|c|}
\hline \multicolumn{1}{|c|}{ Bundesländer } & \multicolumn{1}{|c|}{ Justizvollzugsanstalten } & \multicolumn{1}{c|}{$\begin{array}{c}\text { Anzahl der } \\
\text { Hausfrauenfreigänge 2007 }\end{array}$} \\
\hline Baden-Württemberg & Schwäbisch Gmünd, Bühl, Ravensburg, Heidelberg & 7 \\
\hline Berlin & Berlin, Bereich Reinickendorf & 7 \\
\hline Bremen & Bremen, Außenstelle „Am Fuchsberg “ & 2 \\
\hline Hessen & Frankfurt am Main III, Kassel III (Abteilung Baunatal) & 3 \\
\hline Niedersachsen & Hannover (Freigängerhaus), Vechta (Abteilung Falkenrott) & 2 \\
\hline Nordrhein-Westfalen & Gelsenkirchen, Bielefeld-Brackwede II, Willich II & 15 \\
\hline Rheinland-Pfalz & Koblenz, Rohrbach, Zweibrücken & -16 \\
\hline Schleswig-Holstein & Lübeck & \multicolumn{2}{|c|}{} \\
\hline
\end{tabular}




\section{Literaturverzeichnis}

Birtsch, Vera; Rosenkranz, Joachim (Hrsg.): Mütter und Kinder im Gefängnis. Orientierung und Ergebnisse zum Frauenstrafvollzug und $\mathrm{zu}$ Mutter-Kind-Einrichtungen im Strafvollzug, Weinheim 1988.

Bundesarbeitsgemeinschaft für Straffälligenbilfe e.V. (Hrsg.): Straffälligenhilfebericht 1997/1998. Straffällig gewordene Frauen Lebenslagen und Hilfeangebote, Bonn 1998. (zit. BAG-S e.V. 1998)

Drenkhahn, Kristin: Sozialtherapeutischer Strafvollzug in Deutschland, Mönchengladbach 2007.

Dünkel, Frieder; Geng, Bernd: Fakten zur Überbelegung im Strafvollzug und Wege zur Reduzierung von Gefangenenraten, in: Neue Kriminalpolitik, H. 4/2003, S. 146-149.

Egg, Rudolf: Sozialtherapeutische Anstalten und Abteilungen im Justizvollzug. Mindestanforderungen an Organisation und Ausstattung Indikationen zur Verlegung, in: Forum Strafvollzug, H.3/2007, S.100-103.

Franze, Karin: Resozialisierung unter den Bedingungen des Frauenstrafvollzugs, Frankfurt am Main 2001.

Funk, Ina: Täterinnen und Bestrafte - eine kriminalstatistische Analyse der Frauenkriminalität und aktuelle Bestandsaufnahme des Frauenstrafvollzugs in Deutschland, unveröff. Diplomarbeit an der Fakultät Sozialwissenschaften der Georg-Simon-Ohm-Hochschule Nürnberg, Nürnberg 2008.

Geiger, Manfred; Steinert, Erika: Straffällige Frauen und das Konzept der „Durchgehenden sozialen Hilfe“, Stuttgart 1993.

Grote-Kux, Gabriele: Frauenstrafvollzug in der BRD, Berlin 2007, abrufbar unter http://prisonportal.informatik.uni-bremen.de/prisonportal/index.php/Frauenstrafvollzug_in_der BRD (zit. Grote-Kux 2007 a)

Grote-Kux, Gabriele: Strukturelle Benachteiligung von Frauen und Mädchen im Strafvollzug, Berlin 2007, abrufbar unter http://prisonportal. informatik.uni-bremen.de/prisonportal/index.php/Strukturelle_Benachteiligung_von Frauen_und_M\%C3\%A4dchen_im_Strafvollzug. (zit. Grote-Kux 2007 b).

Hüdepohl, Sabine: Sozialtherapie - ein Konzept auch für jugendliche Intensivtäterinnen?, in: Kawamura-Reindl, Gabriele / Halbhuber-Gassner, Lydia / Wichmann, Cornelius (Hrsg.): Gender Mainstreaming - ein Konzept für die Straffälligenhilfe, Freiburg im Breisgau 2007, S.232-245

Jansen, Irmgard: Mädchen in Haft. Devianzpädagogische Konzepte, Opladen 1999.

Kawamura-Reindl, Gabriele: Straffällige Frauen, in: Cornel, Heinz / Kawamura-Reindl, Gabriele / Maelicke, Bernd / Sonnen, Bernd-Rüdiger (Hrsg.): Resozialisierung - Handbuch, Baden-Baden 2009, S. 344-373.

Kawamura-Reindl, Gabriele; von den Driesch, Danielle: Straffällige Frauen - Lebenslagen und Hilfeangebote, in: Cornel, Heinz (Hrsg.): Neue Kriminalpolitik und Soziale Arbeit, Baden-Baden 2002, S. 179-187.

Maelicke, Hannelore: Ist Frauenstrafvollzug Männersache? Eine kritische Bestandsaufnahme des Frauenstrafvollzugs in den Ländern der Bundesrepublik Deutschland, Baden-Baden 1995.

Obermöller, Bernd: Reform des Frauenstrafvollzugs durch problemorientierte Rechtsanwendung, Baden-Baden 2000

Rehn, Gerhard: Sozialtherapie - Anspruch und Wirklichkeit 2003, in: Neue Kriminalpolitik H.3/2003, S. 66-69.

Simmedinger, Renate: Resozialisierung von straffälligen Frauen, in: Cornel Heinz / Kawamura-Reindl, Gabriele / Maelike, Bernd / Sonnen,
Bernd-Rüdiger (Hrsg.): Handbuch der Resozialisierung, Baden-Baden 1995, S. 195-220.

Spöhr, Melanie: Sozialtherapie im Strafvollzug 2007 - Ergebnisübersicht zur Stichtagserhebung zum 31.03.2007, Wiesbaden 2007, abrufbar unter

http://www.krimz.de/fileadmin/dateiablage/forschung/texte/Sozialtherapie_im_Strafvoll zug_2007.pdf.

Stascheit, Ulrich (Hrsg.): Nomos Gesetze - Gesetze für Sozialberufe, Baden-Baden 2007.

Statistisches Bundesamt (Hrsg.): Bewährungshilfe 2006, Fachserie 10 Reihe 5, Wiesbaden 2007, abrufbar unter https://www-ec.destatis.de/ csp/shop/sfg/bpm.html.cms.cBroker.cls?cmsp ath=struktur,vollanzeige. $\operatorname{csp} \& I D=1021288$. (zit. Bewährungshilfestatistik 2006)

Statistisches Bundesamt (Hrsg.): Bestand der Gefangenen und Verwahrten in den deutschen Justizvollzugsanstalten nach ihrer Unterbringung auf Haftplätze des geschlossenen und offenen Vollzuges jeweils zu den Stichtagen 31.März, 31.August und 30.November eines Jahres, Wiesbaden 2007, abrufbar unter https://www-ec.destatis.de/csp/shop/sfg/bpm. html.cms.cBroker.cls? $\mathrm{cmspath=struktur,volla}$ nzeige.csp\&ID=1019821. (zit. Strafvollzugsstatistik 2007 a)

Statistisches Bundesamt (Hrsg.): Strafvollzug Demographische und kriminologische Merk male der Strafgefangenen am 31.03.2007, Fachserie 10 Reihe 4.1, Wiesbaden 2007, abrufbar unter https://www-ec.destatis.de/ $\mathrm{csp} / \mathrm{shop} / \mathrm{sfg} / \mathrm{bpm}$. html.cms.cBroker.cls?cmsp ath $=$ struktur,vollanzeige $. c s p \& I D=1022190$. (zit. Strafvollzugsstatistik 2007 b)

Stöckle-Niklas, Claudia: Das Gefängnis. Eine eingeschlechtliche Institution, Bonn 1989

Zolondek, Juliane: Lebens- und Haftbedingungen im deutschen und europäischen Frauenstrafvollzug, Mönchengladbach 2007.

InaFunk Ina-Funk@gmx.de arbeitet als Dipl.-Sozialpädagogin in Nürnberg.

\section{Fußnoten:}

Aktuell werden Frauen in 27 abgetrennten Abteilungen, in 16 eigenen Häusern und in acht Außenstellen untergebracht.

2 Darunter fallen Straftaten gegen die sexuelle Selbstbestimmung, Straftaten gegen das Leben, die körperliche Unversehrtheit, die persönliche Freiheit, sonstige Straftaten gegen die Person sowie Raub, Erpressung und räuberischer Angriff auf Kraftfah- rer.

3 Zu Eigentums- und Vermögensdelikte zählen Diebstahl und Unterschlagung, Begünstigung und Hehlerei, Betrug und Untreue, Urkundenfälschung und sonstige Straftaten gegen das Vermögen.

4 Mecklenburg-Vorpommern weist zwar ebenfalls eine Belegungsquote von über $100 \%$ auf. Da es sich allerdings nur um 37 Frauen handelt, die auf 35 Haftplätze untergebracht sind, kann nicht von einer prekären Überbelegungssituation ausgegangen werden.

5 Die Belegungsquoten des Saarlandes und die von Thüringen sind in diesem Zusammenhang zu vernachlässigen, da die meisten Frauen dieser Bundesländer im Rahmen von Vollzugsgemeinschaften in Justizvollzugsanstalten anderes Bundesländer untergebracht sind.

6 Wie bereits erwähnt, verfügt MecklenburgVorpommern über keine Haftplätze im of fenen Vollzug. Saarländische Frauen sind in der JVA Zweibrücken (Niedersachsen) untergebracht. Thüringen hält für Frauen lediglich 12 Haftplätze des offenen Vollzugs bereit. Die übrigen weiblichen Gefangenen sind in der JVA Chemnitz (Sachsen) inhaftiert.

7 Hierbei handelt es sich allerdings um eine Haftanstalt, in der auch männliche Jugendliche bzw. Heranwachsende inhaftiert sind.

8 Die einzige Unterbringungsmöglichkeit für weibliche Jugendliche besteht in der JVA Berlin. Hier können bis zu drei Mädchen aufgenommen werden. (Hüdepohl 2007, S. 232)

9 In der JVA Berlin - Neukölln stehen 18 Haftplätze für die Sozialtherapie, in der JVA Vechta/Teilanstalt Alfeld 11 und in der JVA Dresden 9 Plätze zur Verfügung.

10 In der JVA Berlin-Neukölln wurden 15, in der Teilanstalt Alfeld 10 und in der JVA Dresden 7 weibliche Gefangene in den sozialtherapeutischen Abteilungen untergebracht.

11 Die Anzahl der Haftplätze richtet sich in Schwäbisch Gmünd nach der Zahl der Kinder und nicht nach der Anzahl der Mütter. In den übrigen Anstalten bezieht sich die Anzahl der Haftplätze auf die für Mütter zur Verfügung stehende Plätze.

12 Die halboffene Mutter-Kind Abteilung der JVA Schwäbisch Gmünd befindet sich im nicht sicheren Bereich der Anstalt, direkt an einer nur ca. drei Meter hohen Mauer. Ihre Hafträume sind für mehrere Stunden täglich geöffnet, allerdings eingeschränkter als dies im offenen Vollzug die Regel ist.

13 Das bayerische Justizministerium teilte mit, dass seit Herbst 2007 auch sechs Plätze im offenen Vollzug eingerichtet wurden. Seit Oktober 2008 befinden sich darüber hinaus in der JVA Stadelheim zehn Plätze im geschlossenen Vollzug.

14 Bis zum zweiten vollendeten Lebensjahr können Kleinkinder in der JVA Berlin, Standort Reinickendorf, untergebrach werden. In den Haftanstalten Schwäbisch Gmünd, Berlin am Standort Neukölln, Frankfurt am Main III (geschlossener Vollzug), Vechta (geschlossener Vollzug) sowie in der JVA Chemnitz dürfen Kinder das dritte Lebensjahr nicht überschritten haben.

15 In Rheinland-Pfalz ist der Hausfrauenfreigang prinzipiell in allen Anstalten des of fenen Vollzugs (JVA Koblenz, Rohrbach, Zweibrücken) möglich. Das Justizministerium konnte allerdings keine Auskunft über die Zahl der Hausfrauenfreigängerinnen erteilen, da in der statistischen Erfassung keine Unterscheidung zu Frauen, die z.B. im Rahmen des Freigangs einem freien Beschäftigungsverhältnis nachkommen, vorgenommen wird.

16 In der JVA Lübeck ist Hausfrauenfreigang prinzipiell möglich, 2007 wurde hiervon allerdings kein Gebrauch gemacht.

17 Der von Hannelore Maelike durchgeführten Untersuchung ist $\mathrm{zu}$ entnehmen, dass am 31.03.1993 nur 2.625 weibliche Gefangene inhaftiert waren. (vgl. Maelike 1995 , S.41) Am 31.03.1997 waren es bereits 3.097 (vgl. BAG-S e.V. 1998, S.37) und am 31.03.2007 befanden sich 4.068 Frauen in Haft. (vgl. Strafvollzugsstatistik 2007, S.5) 